\title{
Pituitary Metastasis of Thyroid Follicular Adenocarcinoma
}

- Case Report-

\author{
Hidenobu OCHIAI, Shinichi NAKANO, Tomokazu GOYA, \\ Shinichiro WAKISAKA and Kazuo KINOSHITA
}

Department of Neurosurgery, Miyazaki Medical College, Miyazaki

\begin{abstract}
A rare case of pituitary metastasis of thyroid follicular adenocarcinoma occurred in a 62-year-old female manifesting as left retro-orbital pain and diplopia. Computed tomography and magnetic resonance imaging revealed a tumor in the pituitary fossa extending to the left cavernous sinus, sphenoid sinus, and prepontine cistern, destroying the upper portion of the clivus. An asymptomatic thyroid mass, probably malignant, was also found. She also had an incidental small meningioma in the posterior fossa. The pituitary tumor was partially removed, and the thyroid and posterior fossa tumors were totally removed in two operations. Both pituitary and thyroid tumors were verified to be follicular adenocarcinoma. Postoperatively, she developed panhypopituitarism for which cortisol and thyroxine replacement therapy was necessary. Three years after first therapy, she was alive but her symptoms did not improve.
\end{abstract}

Key words: pituitary gland, metastasis, thyroid carcinoma

\section{Introduction}

Thyroid follicular adenocarcinoma often metastasizes to lung and bone, especially to the skull, ribs, and vertebrae. However, metastasis to the pituitary gland is quite rare with only four cases reported previously. ${ }^{1,2,45}$ Here, we describe a case of pituitary metastasis of thyroid follicular adenocarcinoma.

\section{Case Report}

A 62-year-old female was admitted to our department with diplopia and left retro-orbital pain. Physical examination revealed an elastic hard thyroid tumor in the right lobe, without cervical lymphnode swelling. Neurological examination found left abducens and oculomotor nerve paralyses. Visual acuity and fields were normal. Endocrinological examination revealed no pituitary dysfunction.

Computed tomographic (CT) scans disclosed and

Received October 17, 1991; Accepted May 7, 1992 irregular-shaped, homogeneously enhanced mass in the sella turcica extending to the left cavernous sinus, sphenoid sinus, and prepontine cistern. A round, homogeneously enhanced mass was also found incidentally in the left posterior fossa (Fig. 1). Mag-
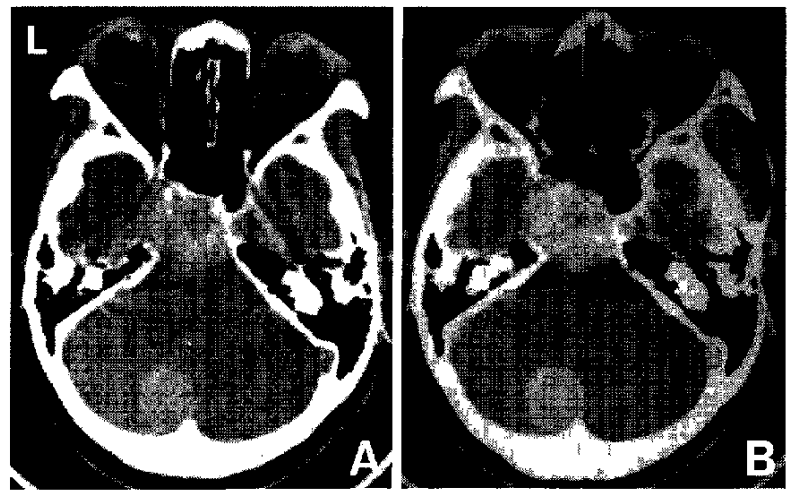

Fig. 1 A: Precontrast CT scan, revealing slightly hyperdense masses in the pituitary and left posterior fossa. B: Postcontrast CT scan, revealing homogeneous enhancement of both tumors. 

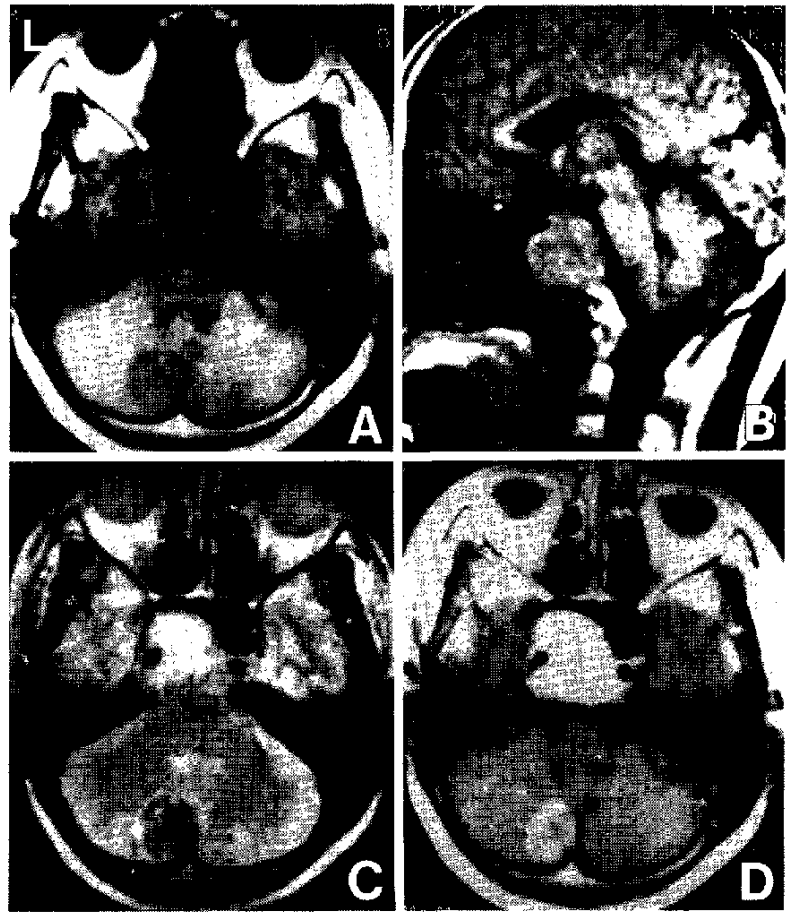

Fig. 2 A: $\mathrm{T}_{1}$-weighted axial MR image (TR/TE, 500/ $30 \mathrm{msec}$ ), showing both pituitary and left posterior fossa tumors as hypointense masses. B: $T_{1}$-weighted sagittal $M R$ image $(500 / 30$ msec), showing pituitary tumor extending to the sphenoid sinus and prepontine cistern. C: $T_{2}$-weighted axial MR image (2000/80 msec), showing pituitary tumor as hyperintense and left posterior fossa tumor as hypointense masses. D: Both tumors are homogeneously enhanced with Gd-DTPA.

netic resonance (MR) imaging using a 0.22 -T scanner showed a hypointense tumor in the pituitary region destroying the upper portion of the clivus on the $\mathrm{T}_{1}$-weighted image (TR/TE, 500/30 msec) (Fig. $2 A, B)$. The tumor was hyperintense on the $T_{2^{-}}$ weighted image (2000/80 msec), and homogeneously enhanced with gadolinium-diethylenetriaminepentaacetic acid (Gd-DTPA) (Fig. 2C, D). Left common carotid angiograms showed a hypervascular tumor fed by the maxillary and meningohypophyseal arteries (Fig. 3). The thyroid tumor appeared as a cold area on technetium- $99 \mathrm{~m}$ scintigrams and a hot area on thallium-201 scintigrams, suggesting a malignant neoplasm. These findings suggested the sellar lesion to be a metastasis from the thyroid cancer.

At the first surgery, the transsphenoidal approach was employed. The tumor had protruded into the sphenoid sinus, covered by paper-thin sellar floor and dura mater. The pinkish-yellow tumor was ex-

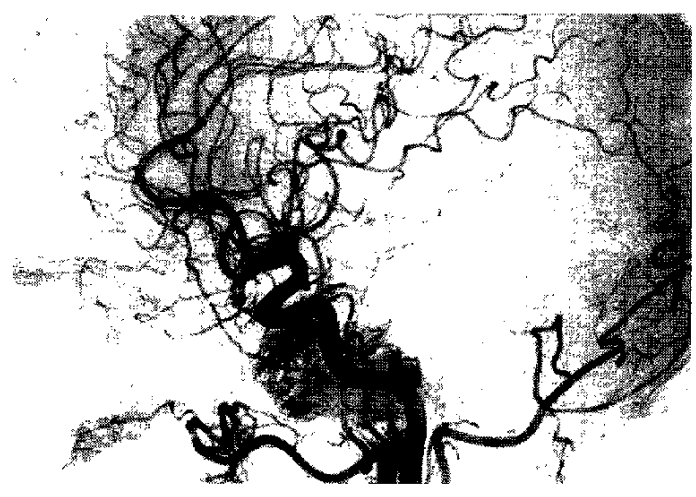

Fig. 3 Left common carotid angiogram, showing the hypervascular pituitary tumor fed by the maxillary and meningohypophyseal arteries.

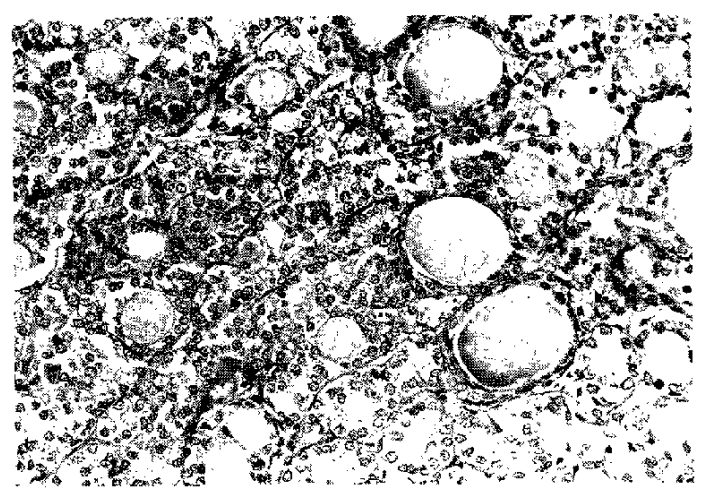

Fig. 4 Photomicrograph of the surgical specimen of the metastatic pituitary tumor, revealing follicular adenocarcinoma.

posed by dural incision. The tumor was parenchymatous and gelatinous, and continued into the pituitary fossa. Only the tumor protruding into the sphenoid sinus was removed and no normal pituitary gland was detected.

Postoperatively, she developed panhypopituitarism and received cortisol and thyroxine replacement therapy. Twenty-four days later, the thyroid and left posterior fossa tumors were removed totally. After this, internal irradiation using ${ }^{131} I$ was performed against the residual tumor of pituitary region and she discharged after this therapy. But the residual tumor did not reduce its size and her symptoms did not improve. Three years after first therapy, she was alive and well, but left abducens and oculomotor nerve paralyses were still unchanged and the replacement therapy of thyroxine and cortisol was still necessary.

Histological examination of the pituitary tumor 
Table 1 Reported cases of symptomatic metastatic pituitary tumor from thyroid carcinoma

\begin{tabular}{|c|c|c|c|c|}
\hline Author (Year) & Histological type & Cranial nerve palsy & Pituitary dysfunction & Sellar change \\
\hline $\begin{array}{l}\text { Johnson and Atkins } \\
(1965)^{1)}\end{array}$ & - & oculomotor, trochlear & - & erosion \\
\hline $\begin{array}{l}\text { Kistler and Pribram } \\
(1975)^{2)}\end{array}$ & $\begin{array}{l}\text { follicular } \\
\text { adenocarcinoma }\end{array}$ & oculomotor & - & destruction \\
\hline Sanchez et al. $(1977)^{4)}$ & - & oculomotor, trochlear & - & erosion \\
\hline Sziklas et al. $(1985)^{5}$ & $\begin{array}{l}\text { papillary } \\
\text { adenocarcinoma }\end{array}$ & - & hypopituitarism & demineralized \\
\hline Present case & $\begin{array}{l}\text { follicular } \\
\quad \text { adenocarcinoma }\end{array}$ & oculomotor, abducens & - & destruction \\
\hline
\end{tabular}

revealed characteristic features of thyroid follicular adenocarcinoma. Polygonal or cubital cells were arranged in a tubular or follicular pattern containing colloid materials, showing invasive growth into the fibrous stroma (Fig. 4). The thyroid tumor was also a follicular adenocarcinoma and the posterior fossa tumor was a fibroblastic meningioma.

\section{Discussion}

Metastatic pituitary tumor is unlikely to be recognized clinically, but the incidence is as high as 6$25 \%$ in autopsy cases of carcinoma. ${ }^{3)}$ The breast and lung are the most frequent primary sites in females and males, respectively. ${ }^{3)}$ In contrast, symptomatic pituitary metastasis from thyroid cancer is quite rare with only four such cases reported..$^{1,2,4,5)}$ Three manifested as oculomotor and/or abducens nerve paralyses, as in our case (Table 1).

Metastatic pituitary tumor occurs in the posterior lobe in about $56.8 \%$ of cases because of the characteristic blood supply. Therefore, metastatic pituitary tumor tends to cause diabetes insipidus rather than hypopituitarism. ${ }^{6)}$ However, only $6.8 \%$ of cases showed diabetes insipidus and adenohypophyseal failure is quite rare. Therefore, metastatic pituitary tumor from thyroid cancer, which slowly grows extensively rather than invasively, may manifest as adjacent nerve paralysis, rather than pituitary dysfunction.

We could not determine the exact metastatic site preoperatively, but intraoperative findings disclosed that the surface of the tumor protruding into the sphenoid sinus was covered with paper-thin sellar floor and dura mater, indicating that the tumor was present in the intradural space. Teears and Silverman ${ }^{6}$ reported that no case involved the pituitary gland by adjacent osseous metastasis. Therefore, the metastatic site in this case was probably the pituitary gland.
There are three possible pathways for metastasis to the pituitary gland: 1) hematogenous spread to the gland parenchyma, 2) extension from juxtasellar and skull base metastases, and 3 ) invasion of the suprasellar cistern by leptomeningeal tumor. In our case, the sellar and juxtasellar bony metastasis was not considerable. Furthermore, we could not detect leptomeningeal tumor invasion of the suprasellar cistern by preoperative MR imaging. The metastatic pathway in our case was therefore hematogenous spread to the gland parenchyma.

Recent progress in the treatment of carcinoma has achieved longer survival. As a result, the incidence of metastatic brain tumor has been increasing. Likewise, the incidence of symptomatic metastatic pituitary tumor may also increase.

\section{References}

1) Johnson PM, Atkins HL: Functioning metastasis of thyroid carcinoma in the sella turcica. J Clin Endocr 25: 1126-1130, 1965

2) Kistler M, Pribram HW: Metastatic disease of the sella turcica. $A J R$ 123: 13-21, 1975

3) Roessmann U, Kaufmann B, Friede RL: Metastatic lesion in the sella turcica and pituitary gland. Cancer 25: 478-480, 1970

4) Sanchez JA, Rahman S, Strauss RA, Kaye GI: Multiple myeloma masquerading as a pituitary tumor. Trans Ophthalmol Soc UK 102: 481-486, 1977

5) Sziklas JJ, Mathews J, Spencer RP, Rosenberg RJ, Ergin MT, Bower BF: Thyroid carcinoma metastatic to pituitary. $J$ Nucl Med 26: 1097, 1985

6) Teears RJ, Silverman EM: Clinicopathological review of 88 cases of carcinoma metastatic to pituitary gland. Cancer $36: 216-220,1975$

Address reprint requests to: H. Ochiai, M.D., Department of Neurosurgery, Miyazaki Medical College, 5200 Kihara, Kiyotake-cho, Miyazaki-gun, Miyazaki 88916, Japan. 